The Theory of Order-Disorder in Alloys

By M. A. Krivoglaz and A. Smirnov. Pp. $x+427$. (London: Macdonald and Co. (Publishers), Ltd., 1964.) $90 s$.

$\mathrm{N}$ an ordinary metallic solid solution of, say, a twocomponent system, the two species of atoms are arranged at random on the points of the crystal lattice, and at a composition $A B$, for example, any particular lattice point may be occupied by either $A$ or $B$ atoms. In certain systems, however, below a critical temperature, segregation of one kind of atom to a certain set of lattice points can occur, leaving the other kind to the remaining positions. This phenomenon is known as 'ordering'. The possibility of ordering had been suggested by Tammann in 1919 , and was confirmed by the X-ray work of Bain in 1923. Since that time many metallic systems have been discovered to have this property at certain simple ratios of $A$ to $B$, the best-known examples occurring at compositions $A B$ and $A_{3} B$, such as $\mathrm{Cu}_{3} \mathrm{Au}$ and $\mathrm{AgZn}$. Clearly such a modification of the structure of the alloy as the temperature falls is expected to influence in turn many of the physical and mechanical properties, and a considerable theoretical structure has been erected to relate the observed changes in properties to changes in the models of the electronic structure.

The present volume is a translation from the Russian, and the approach is largely theoretical. It consists of eight chapters. The first chapter is a general review of the properties of ordered alloys, and the second, third and fourth deal with the thermodynamic and statistical theories of ordering, and with the theory of diffusion. A discussion of the effect of ordering on the behaviour of the valence electrons appears in chapter five, and the effect of ordering on wave-motion in chapter six. Chapters seven and eight cover the theory of residual resistivity and the magnetic, galvanomagnetic, optical and mechanical properties of metals and alloys.

The book is aimed at graduate and post-graduate workers and is a fairly comprehensive treatise on the theoretical aspects of ordering. However, there are a number of points of criticism. The treatment is excessively formal, and the explanation of the relation between the mathematical and physical models is disappointing. The first chapter of ninety or so pages is well illustrated with eighty-six figures, but the remaining three hundred pages have only twenty-eight illustrations between them. It is pleasant to have many references to Russian work in the text, but the overall balance of the book suffers. For example, in the chapter on the theory of the residual resistivity of alloys the names of Mott and Friedel do not appear. The book has three hundred and thirty-seven references, but since it was published in Russian in 1958 the most recent reference is 1957. In the copy sent to me for review the quality of the printing and the pages was somewhat uneven. There is no index, and in view of the price one can make only a cautious recommendation.

\section{J. A. Cattherall}

\section{Ultrasonic Cutting}

By L. D. Rozenberg, V. F. Kazantsev, L. O. Makarov and D. F. Yakhimovich. Translated from the Russian by J. E. S. Bradley. Pp. xi +142 . (New York: Consultants Bureau, 1964.) 17.50 dollars.

7 HIS carefully translated text is based primarily on the experience of the authors in the Ultrasonic Laboratory at the Acoustics Institute of the Academy of Sciences of the U.S.S.R., and in the Special Designs Office of the Moscow City Economic Council. The authors have, however, attempted to make due note of work done elsewhere-both inside and outside the U.S.S.R.-and the inclusion of more than 220 literature references is ono result of this.

Since this book should be just as useful in creating interest in, and potential users for, this technique, as in providing a reference text for those already expert, it may be as well to give a brief reminder of what ultrasonic cutting is. It is a manufacturing procoss that has developed over the past twenty years to facilitate the cutting of, or drilling of holes in, materials normally too brittle for working by conventional methods. A slurry containing extremely hard abrasive particles (for example, 'Carborundum' or boron carbide) is fed to the working position, where an acoustically designed tool of relatively soft material vibrates at ultrasonic frequency, hammering down on the trapped particles. In this way an extremely rapid succession of intense 'micro-blows' is imparted to the working surface which thus erodes away, loaving a cavity which reflects the shape of the acoustic tool. Accordingly, and depending on the manner in which the tool is vibrated (for torsional vibrations have as much usefulness as longitudinal vibrations), it is possible to produce round holes, non-circular holes, twisted holes, slots, curved holes, disks and internal and external screw threads, among other forms. Versatility is limited only by the nature of the workpiece material, which must be brittle.

The text covers basic principles as well as practical development. The first chapter discusses elastic wave propagation and vibration for various media, both solid and fluid. Subsequent chapters show how these concepts lead to a detailed understanding of ultrasonic machining, so that-making use of piezo-electric or magnetostrictive excitation-the acoustic tool head may be logically designed to achieve the desired effect. Discussion thereafter widens to deal with different types of ultrasonic machine tool, and indeed with the whole technology of the process.

The book is remarkably readable: the mathematical analysis employed in the early chapters is a digestible minimum and is well presented. Apart from the numerous references already mentioned, the text is finally supported by 31 well-chosen tables of numerical data, and 155 clear illustrations.

B. N. Cole

\section{Contributions to Sensory Physiology}

Vol. 1. Edited by William D. Neff. Pp. $x+274$. (New York: Academic Press, Inc.; London: Academic Press, Inc. (London), Ltd., 1965.) $60 s$.

THIS is the first volume in a new series of publications which has two main objectives, to bring togeth er reports of current research on sensory physiology, and to enable individual research workers to present some particular theory of the physiological basis of sensation. They are definitely not intended to be review articles.

Of the five articles which it contains, three are concerned with vestibular function. The first is a study of the organ of Corti at a cellular level, another concerns the functional anatomy of the ciliated end organs of the labyrinth and lateral line and the third is a psychophysiological study of vestibular responses. If the editors continue to bring together articles like these, which are not normally found in the same volume, the series will certainly fulfil a useful function.

The remaining two articles are on vision and are of particular interest from the comparative point of view as one gives an account of work, some unpublished, on the vision of various primates, while the second is a translation from the French of a paper by Piéron on vision in intermittent light. It is a sad fact, but nevertheless true, that many English-speaking biologists have little time to digest the details of longer papers written in other languages. The publication of translations of this type could do a great deal to help in the dissemination of results that otherwise might not be read in the detail they deserve.

The whole volume is well bound, and has a good reference list and author and subject indexes. It is only unfortunate that the price is so high for it adds to the everincreasing burden that libraries must bear in subsidizing the costly production of volumes of this type.

G. M. HuGHES 